\title{
Evolutionary Optimization of Building Facade Form for Energy and Comfort in Urban Environment through BIM and Algorithmic Modeling
}

\section{A case study in Porto, Portugal}

\author{
Pedro Santiago ${ }^{1}$ \\ ${ }^{1}$ Fernando Pessoa University \\ ${ }^{1}$ psantiag@ufp.edu.pt
}

Consolidated urban areas usually present a challenge for the sustainable design decisions for the architect. The site, orientation and surrounding built environment compromise both passive and active systems, shortening the possible optimization measures available, leaving the designer with doubts as far as efficiency is concerned.BIM methodologies and visual programming languages have opened up a very wide range of design and analysis tools allowing the architect to make informed decisions based on data extracted from the models. Nonetheless it's optimization is through a slow process of trial and error, creating a significant limitation. This paper discusses the potentialities of the use of evolutionary algorithms to generate optimized solutions for facade solar orientation. A comparison between three different evolutionary algorithms aiming for solar radiation, inside average temperature allows to conclude the best result versus time consumed. Although under similar results the multi-objective EA represents the best compromise between time and final objective on the case study chosen for the paper. The interconnectivity in real time of BIM and algorithmic modeling softwares represents an advantage for time saving sustainable design decisions.

Keywords: BIM, Evolutionary Optimization, Sustainable design

\begin{abstract}
1. INTRODUCTION
Among the various strategies to improve energy efficiency in Europe, buildings should be considered as one of the main objectives as they account for about $40 \%$ of energy consumption in Europe. Current energy efficiency directives in EU buildings (Directives 2002/91 / EC, 2010/31 / EU, Nearly Zero Energy Build-
\end{abstract}

ing) impose new requirements on the construction and renovation of buildings with the aim of creating a NZEB built-up landscape. In this premise the approach to the project will have to resort to informed strategies based on concrete and realistic data. The adoption of intelligent strategies will be essential to optimize built envelopes and can start from the 
form factor in order to minimize energy consumption while ensuring the comfort of its occupants. In the context of own generation of energy from renewable sources, in order to reach the closest point of the zero energy balance, an antagonistic problem of maximizing solar gains (the most abundant energy source) is generated, in parallel with the minimization of gains in the hottest periods of the year.

Solar gains can contribute positively to the thermal energy performance of the building, in the form of passive gains, or negatively, in case of need due to lack of exposure or formal and material lack of control. Direct solar radiation received is able to increase comfort inside the building during the cold months and simultaneously decrease the need for heating. However, during the summer the opposite effect occurs, since solar radiation can cause overheating and thus increase the need for cooling.

If this situation is complex in an isolated context, the urban landscape, with the surrounding built environment presents a greater challenge. If we consider that half of the world's population lives in cities that collectively consume three quarters of global resources, with predictions of their increase to three quarters by 2050 [1], it is imperative to understand and create strategies to minimize energy consumption in this particular environment. The approach of maximizing the use of ambient solar energy whether for the active conversion using solar thermal collectors and / or photovoltaic or by bioclimatic passive strategies, allows the reduction of the need of energy for heating and lighting. To work towards this goal, computational modeling directed to the availability of solar radiation can be a tool to support design decisions for architecture and urbanism. However the probability of finding an ideal solution for the geometric shape of the building by manual trial and error method is extremely small.

Calculations can be made for determining the solar envelope on paper using the solar azimuth and elevation data alongside the key heights of the year. However, paper calculations are a long and inaccurate process for urban environments. Nonetheless, nowadays many computer-aided and parametric design programs integrate environmental simulation tools that include the ability to evaluate solar envelopes automatically. It is a simple procedure that requires little external information, such as location, surrounding volumes, plans that define the area to be evaluated and periods of analysis. However, this system only allows to evaluate the potential of the solar envelope, being the process of manual optimization, often based on the experience and knowledge acquired from the designer, limiting the range of solutions to these factors and the time available for the design. Given the geometric and volumetric complexity of the urban environment along with the objectives of the study, an alternative and more efficient method is proposed.

This paper proposes a method to generate solar envelopes that consider multi-directional requirements for access to direct solar gain in complex urban environments. This method was developed for the evaluation of the potential of construction in existing areas in the city of Porto, integrating calculations of solar direct access from environmental computational simulations, generation of solar envelopes using parametric design and optimization plugins. The urban model to integrate the proposal was built in a BIM software, allowing real-time connection with the visual programming tool where the algorithm was developed. The environmental simulation tool used to calculate the hours of direct sun exposure in Grasshopper is Ladybug [2], based on its sun-path Radiance component, a validated tool for solar radiation simulation [2]. The optimization plugins used are Galapagos [3], Opossum and Octopus that allows the application of the principles of evolutionary and model-based optimization. 
Figure 1

Site aerial view.

Figure 2

Street view.

Figure 3

Birdseye view.

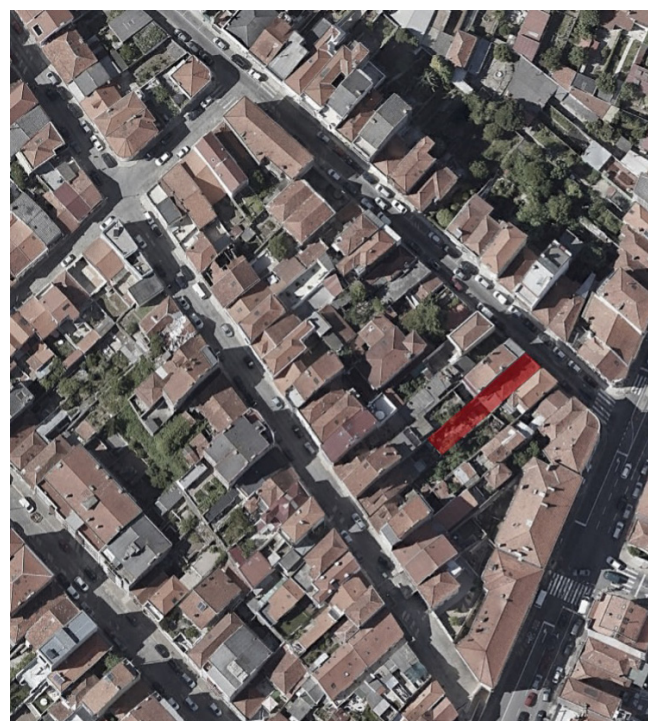

\section{METHODOLOGY}

The described method is applied to a building situated in a consolidated urban context of the city of Porto.

The direct surroundings represent two residential buildings. To the north the volume rises to 4 storeys and to the south to 3 storeys. The volumes on the opposite side of the block are located at a height of 2 floors above the front of the building to be analyzed.

Thus, the patio areas have walls of 6 meters high preventing solar access. Both have inclined roofs of two and four faces. The orientation of the plots is on the northeast-southwest axis, representing an unfavourable orientation from the perspective of gains and solar control, given the predominance of sun with a low angle corresponding to east and west. The number of hours of exposure on the façade facing the street is scarce due to two factors: orientation and direct surroundings. The rear façade is also very conditioned by the reasons already mentioned. The footprint area will be maintained, and the alignment with the respective neighboring volumes allows some formal freedom given the mismatch of limits in the patio and in the roofs.

In a first phase, a study of the solar potential of the site was carried out within a period of one year. The evident or traditional design of alignment and geometry concordances generated a volume that was also subjected to analysis for reference, basis and comparison of results.

The surfaces and volumes generated in BIM environment are then exported to Grasshopper where the analyses are performed on the basis of an algorithm construction. Any changes can be reintroduced into Archicad, which allows you to add information and not its loss throughout the process.

Thus, two periods of the year corresponding to the hot season from June to September and to the temperate and cold season corresponding to the remaining months of the year were considered in order to obtain maximum sun exposure in the temperate and cold months and to have the opposite situa- 


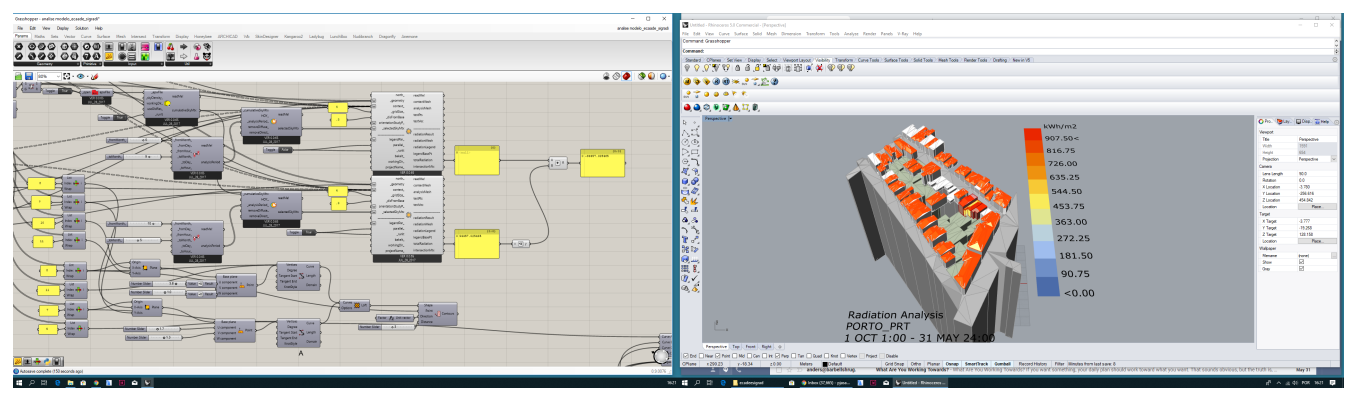

Figure 4

Radiation Analysis.

\begin{tabular}{lccccc}
\hline & $\begin{array}{c}\text { Solar } \\
\text { radiation hot } \\
\text { season (KWh) }\end{array}$ & $\begin{array}{c}\text { Solar radiation } \\
\text { temperate and } \\
\text { cold season } \\
\text { (KWh) }\end{array}$ & $\begin{array}{c}\text { Total } \\
\text { radiation } \\
\text { (KWh) }\end{array}$ & Difference & $\begin{array}{c}\text { Time spent } \\
\text { to calculate }\end{array}$ \\
\hline Base building & 72267,9 & 93357 & 165624,9 & 21089,10 & \\
Galapagos & 69548,2 & 98789,8 & 168338 & 29241,60 & $30 \mathrm{~h}$ \\
Opossum & 68317,5 & 99102 & 167419,5 & 30784,50 & $12 \mathrm{~h}$ \\
Octopus & 68950,3 & 100215,3 & 169165,6 & 31265,00 & $18 \mathrm{~h}$ \\
\hline
\end{tabular}

\begin{tabular}{|c|c|c|c|c|}
\hline & Base building & Galapagos & Opossum & Octopus \\
\hline $\operatorname{March} 21^{\text {st }}$ & 16.41 & 18.68 & 18.76 & 19.44 \\
\hline June $21^{\text {st }}$ & 25.03 & 24.91 & 24.88 & 23.03 \\
\hline September $21^{\text {st }}$ & 21.36 & 22.44 & 22.44 & 23.36 \\
\hline December $21^{\text {st }}$ & 13.28 & 14.55 & 14.63 & 15.01 \\
\hline
\end{tabular}

Table 2

Internal average temperatures 
tion in the hot months of the year. The solution will focus only and exclusively on the form factor of the proposed building.

The algorithm allows to control manipulable points from variable values within a limit and direction. The union of the latter generates lines whose union in turn generates surfaces. Since the system is not static, it allows a great degree of freedom and combinations within the alignments imposed by the urban rules. This system is repeated in the roof, allowing the generation of all the skin of the building according to the needs and the respective solar quadrants. In the end those same surfaces will be connected with the native elements of Archicad still as elements of the algorithm allowing the symbiosis of the work systems.

Technical or efficiency problems can be faced or even interpreted as opportunities to improve the architectural quality of a project. Efficiency is increasingly a requirement, especially when it comes to issues of energy consumption and comfort. A building dependent on mechanical systems has energy costs and constant maintenance. Efficiency depends on the approach to design from the first steps of the planning process. This approach is not always compatible with the formal elements resulting from cultural analysis and willingness to materialize from ideas. However, when this process evolves or is developed in parallel it is less likely to come into conflict.

Often the search for form includes efforts of representation where it is refined from drawings and models in the search for results based on inspiration. With this in mind, it is intended to point out efficiency as an element that generates interesting and functional formal solutions.

Evolutionary algorithms are based on natural selection, creating groups of genes that are tested and then crossed among the most adapted, generating optimized solutions. A typical evolutionary algorithm process includes selection, crossover, and mutation. First, a series of points are randomly generated as the initial population. An assessment is then conducted to assess the suitability of each individual. The most suitable individuals for the ideal solution are chosen as parents of the next series or generation by crossover and mutation. The following generations go through the same process repeatedly until the end of the process or algorithm. Compared to the direct search methodology, the evolutionary algorithm has a higher calculation speed, higher accuracy and stronger adaptability. Based on their different implementation details, evolutionary algorithms have been classified as genetic, neuro-evolutionary, particle swarm optimization and other types.

In this case three different evolutionary algorithms were considered. Galapagos, the default EA from grasshopper3d that works with one objective; Opossum, that also works with one objective; and Octopus a multi-objective evolutionary algorithm.

The objective is to compare seasonal radiation, time spent to optimize and internal general building temperatures in key moments of the year, corresponding to the 21 st of March, June, September and December, of each solution given by the different $E A$.

In construction terms, the systems used were those described below.

The slab consists of $25 \mathrm{~cm}$ of solid concrete slab with $6 \mathrm{~cm}$ thermal insulation, $5 \mathrm{~cm}$ regularization layer and vinyl finishing.

This system has a $U$ value of $0.54 \mathrm{~W} / \mathrm{m} 2 \mathrm{~K}$.

The wall corresponding to the facade consists of an external plastered insulation system whose support is a thermal ceramic block plastered by the interior.

This system presents a $U$ value of $0.26 \mathrm{~W} / \mathrm{m} 2 \mathrm{~K}$.

The roof slab consists of a concrete slab of $25 \mathrm{~cm}$ isolated superiorly, supporting a suspended ceiling in plasterboard with an air gap of $1 \mathrm{~m}$ in height,

This system has a $U$ value of $0.10 \mathrm{~W} / \mathrm{m} 2 \mathrm{~K}$.

For the glazed spans, metal frames in aluminium with thermal separation with a $U$ value of 1.8 corresponding to the nomenclature "premium" and a double glazing without any solar factor with a $U$ value of 1.7 were considered.

This system has a $\mathrm{U}$ value of $2.05 \mathrm{~W} / \mathrm{m} 2 \mathrm{~K}$. 
Points of wall and roof construction were created, which can be manipulated from variable values within maximum and minimum limits and direction. The union of the latter generates lines whose union in turn generates surfaces. Since the system is not static, it allows a great degree of freedom and combinations within the alignments imposed by the urban rules. This system intends to create surfaces that contain constructive elements allowing to change their angle in relation to the solar incidence. They also allow changing their height in the case of roofs, and can be optimized according to the needs and solar quadrants. Throughout the process, these elements are connected to the native elements of Archicad as well as elements of the algorithm allowing the symbiosis of the work systems.

Multi-objective optimisation is based on incident radiation on each surface in order to obtain maximum sun exposure in the temperate and cold months and to have the opposite situation in the hot months of the year. The optimization solution will focus on the shape, angle and size of the surfaces of the southwest quadrant of the proposed building.

The temperature reading will be from the ecodesigner star, without any system assigned to each compartment, i.e. in an absolutely natural/bioclimatic way.

\section{RESULTS}

The base solution presents a total annual radiation of $165624,9 \mathrm{kw} / \mathrm{h}$ distributed by $72267,9 \mathrm{kw} / \mathrm{h}$ in the hot months and the remaining $93357 \mathrm{kw} / \mathrm{h}$ in the temperate cold months of the year. Despite these absolute results, it can be verified by the volume geometry the presence of some shaded areas, which creates little uniformity in the distribution of solar energy.

The solutions proposed by the algorithm's calculation results present a higher annual total radiation, which may lead to a negative conclusion of the process.

An analysis by each evolutionary algorithm, however, reveals less solar incidence in the hot months and more throughout the rest of the year.
The energy potential is higher and distributed according to the needs of the building.

The results are similar on all EAs. The formal final results are also similar, probably because the freedom degree was not too permissive. The differences on the radiation results are not very substantial.

The fastest EA was Opossum and the slowest was Galapagus. Both are single objective. The one that performed better in this particular case was Octopus, with better indoor temperature results and an intermediate calculation time.

\section{CONCLUSIONS}

The tools and methodology presented, offer the architect an improved and clearer access to the results of multi-objective optimization along with the ability to classify, filter, comprehend the formal aspect of the solution, considering the overall performance of the project. Archicad provided a common platform for additional functionality in building design development by combining multiple different domains by connecting to Grasshopper3d and its sub products such as Galapagos, Opossum, Octopus and Ladybug, according to the architect's cognitive preferences and skills. When Grasshopper3d changes its platform to support 64-bit computing and parallel processing, it will increase the speed of these slow processes.

The potential of the described workflow is significant at the beginning of project development and can be even more efficient, intelligent and flexible. The intention is to provide the architect with the ability to deal with an increasingly demanding field of complexity in a clear and visual manner, always based on data and design.

One of the main objectives of the research described in this article was to reduce the distance and error inherent to the migration of information between various platforms in the design cycle through the integration of parametric design cycles in real time, multiobjective optimization, simulation and return. By holistically integrating the programs and automating the workflow using a common platform, 
some of the frequently encountered interpretation and data exchange problems were avoided.

As demonstrated in this case study, the suggested models not only offer good solutions, but also contribute to a better understanding of design problems. The possibility of examining the same data set with different assessment schemes allows one to face and rethink the systems and methodologies inherent to architectural design problems. In other words, optimization models are a promising starting point for the development of generative design tools or for the exploration of informed solutions and the establishment of new relationships between the architect and the computer and help to generate and visualize design knowledge in the form of models informed by concrete data.

The design and the project informed by data often implies the use of several programs with very distant methodologies that create alienation of the initial concepts. The migration of information between programs causes losses and distortions of results. The proposed method takes advantage of the capabilities of several programs without leaving the base program, in this case Archicad. The BIM methodology did not allow in an isolated way to incorporate the advantages and capacities of generation of solutions made possible by the programs of construction of parametric and generative algorithms, along with all the coupled subprograms.

The advantage of the proposed method is the real-time permission, a very important factor in the development of the work process in the initial phase of the project, to constitute a work system that goes through the concept implementation process, its analysis and optimization processes, in this case using evolutionary processes. The examples presented always refer to digital environments external to the base design program, which always compromises the agility of thinking and analysis processes, along with errors in importing and exporting and sometimes data loss. This proposal is integral, dynamic and manages to harness the advantages of both systems. The result is a more advantageous base, allow- ing also the work with more adapted forms without compromising other factors and conditions.

\section{DISCUSSION}

The optimization techniques and systems currently used have several limitations. The design and design informed by data often implies the use of several programs with very distant methodologies that create alienation of the initial concepts. The migration of information between programs causes losses and distortions of results. The proposed method takes advantage of the capabilities of several programs without leaving the base program, in this case Archicad. The BIM methodology did not allow, in isolation, to incorporate the advantages and capabilities of generating solutions made possible by the parametric and generative algorithms construction programs, along with all the coupled subprograms.

The advantage of the proposed method is the real-time permission, a very important factor in the development of the work process in the initial phase of the project, to constitute a work system that goes through the process of implementation of the concept, its analysis and processes of form optimization, in this case with recourse to evolutionary processes. The examples presented always resort to digital environments external to the basic design program, which always compromises the agility of thought and analysis processes, along with export import errors and sometimes data loss. This proposal is integral, dynamic and can leverage the advantages of both systems. The result is more advantageous, allowing also the work with more adapted forms without compromising other factors and constraints.

\section{REFERENCES}

Caruso, G. and Kampf, J. H. 2015, 'Building shape optimisation to reduce air-conditioning needs using constrained evolutionary algorithms', Solar Energy, 118, pp. 186-196

Kampf, J. H. and Robinson, D. 2010, 'Optimisation of building form for solar energy utilisation using constrained evolutionary algorithms', Energy and Buildings, 42, pp. 807-814 
De Luca, F. 2016 'Solar Envelope Optimization Method for Complex Urban Environments"', CAADence 2016, Budapest

United Nations, UN (eds) 2014, World Urbanization Prospects: The 2014 Revision, United Nations

Sadeghipour, M. and Smith, A. 2013 'Ladybug: a parametric environmental plugin for Grasshopper to help designers create an environmentally-conscious design', Proceedings of BS2013: 13th Conference of International Building Performance Simulation Association, pp. 3128-3135

Wright, J. A., Brownlee, A., Mourshed, M. and Wang, M. 2014, 'Multi-objective optimization of cellular fenestration by an evolutionary algorithm', Journal of Building Performance Simulation, 7:1, pp. 33-51

Yi, Y. K. and Hyoungsub, K. 2015, 'Agent-based geometry optimization with Genetic Algorithm (GA) for tal apartment's solar right," Solar Energy, 113, pp. 236250 